\title{
$c$-Jun is required for the specification of joint cell fates
}

\author{
Akinori Kan and Clifford J. Tabin ${ }^{1}$ \\ Department of Genetics, Harvard Medical School, Boston, Massachusetts 02115, USA
}

\begin{abstract}
Joints form within the developing skeleton through the segmentation and cavitation of initially continuous cartilage condensations. However, the molecular pathways controlling joint formation largely remain to be clarified. In particular, while several critical secreted signals have been identified, no transcription factors have yet been described as acting in the early stages of joint formation. Working upstream of the early joint marker Wnt9a, we found that the transcription factor $c$-Jun plays a pivotal role in specifying joint cell fates. We first identified an enhancer upstream of the Wnt9a gene driving joint-specific expression in transgenic reporter mice. A comprehensive in silico screen suggested $c$-Jun as a candidate transcription factor activating this Wnt9a enhancer element. $c$-Jun is specifically expressed in joints during embryonic joint development, and its conditional deletion from early limb bud mesenchyme in mice severely affects both initiation and subsequent differentiation of all limb joints. c-Jun directly regulates Wnt16 as well as Wnt9a during early stages of joint development, causing a decrease of canonical Wnt activity in the joint interzone. Postnatally, $c$-Jun-deficient mice show a range of joint abnormalities, including cartilaginous continuities between juxtaposed skeletal elements, irregular articular surfaces, and hypoplasia of ligaments.
\end{abstract}

[Keywords: c-Jun; Wnt9a; Wnt16; skeletal development; joint formation]

Supplemental material is available for this article.

Received November 19, 2012; revised version accepted February 1, 2013.

Joints are essential for all of our movements, from locomotion to fine motor skills, and their impairment causes significant problems for our quality of life. Indeed, arthritis is increasingly an important social and economic concern as our population ages. In spite of the importance of the joints, the mechanisms by which they are formed during embryonic development have remained poorly understood.

The skeleton of vertebrate limbs forms from an initially continuous condensation of mesenchymal cells. Individual skeletal elements then arise through a segmentation process, introducing joints in specific locations. At a descriptive level, a localized dense region known as an interzone is first formed at the location of the future joint within the mesenchymal condensation, followed by differentiation into articulate cartilage at the ends of what will be two juxtaposed skeletal elements separated by a region of cavitations. The forming joint is ultimately encased in a capsule and synovium in addition to associated ligaments. However, how mesenchymal cells are directed to form an interzone and how the interzone differentiates into distinct joint components are largely unknown. In particular, no transcription factors acting at the early stages of this process have yet been identified.

${ }^{1}$ Corresponding author

E-mail tabin@receptor.med.harvard.edu

Article is online at http://www.genesdev.org/cgi/doi/10.1101/gad.209239.112.
Two genes encoding secreted proteins, Wht9 $a$ and Gdf5, have been shown to play important roles in early joint formation. Wnt9a misexpression can induce expression of a wide range of joint markers, including Gdf5, Autotaxin, and Chordin, in chondrogenic regions (Hartmann and Tabin 2001). However, Wnt9a is not required for joint initiation (Spater et al. 2006b). Embryonic joint formation proceeds normally in Wnt9a-deficient mice, although they show small ectopic cartilage nodules called synovial chondroid metaplasia in the elbow and fail to undergo cavitation of a limited number of carpal joints postnatally. Thus, Wnt9a, although sufficient to induce the jointforming program, is not necessary in vivo for it to occur.

Conversely, Gdf5 is required for the normal formation of a number of joints but is not sufficient to initiate the joint-forming process. A loss-of-function mutation of the Gdf5 gene causes the loss of several joints in mice (Storm et al. 1994). Similarly, a truncated GDF5 protein in humans is known to cause a congenital joint malformation called Grebe-type chondrodysplasia (Basit et al. 2008). However, gain of function of $G d f 5$ does not result in joint formation in the developing limb (Tsumaki et al. 2002). In addition to its developmental role, a single-nucleotide polymorphism in the human GDF5 promoter is associated with susceptibility to osteoarthritis, suggesting an additional role in articular cartilage homeostasis (Miyamoto et al. 2007). 
Although Gdf5 and Wnt9a play roles in joint formation, their loss does not affect all limb joints (Storm and Kingsley 1996; Spater et al. 2006b), suggesting the existence of other molecules operating upstream of these genes, specifying the location of the forming joints. However, as Gdf5 and Wnt9a are among the earliest known markers of joint initiation, their expression can be used as a starting point to work backward to identify factors involved in the earliest steps of joint induction.

\section{Results}

Identification of the joint-specific enhancer in the Wnt9a gene

To identify genes that might act upstream of Wnt9a in joint specification, we first set out to identify the cisregulatory sequences responsible for directing expression of this gene in the early joint primordium. To that end, we searched for phylogenetically conserved DNA sequences in and around the Wnt $9 a$ locus in the mouse, human, rhesus monkey, and horse genomes using the University of California at Santa Cruz (UCSC) genome and VISTA browsers. Two conserved blocks of DNA sequence were identified 5' of the Wnt9a gene (Supplemental Fig. S1). One is a 170-base-pair (bp) region just upstream of the transcription start site, and the other is a 240-bp region located $4 \mathrm{~kb}$ upstream of the transcription start site.

To test whether either of the conserved sequences contained regulatory sequences potentially directing expression in the developing joints, we created reporter constructs with each of the conserved candidate regions placed upstream of an Hsp68 minimal promoter and a LacZ reporter gene. To increase sensitivity in transgenic animals, we used four tandem repeats of each putative regulatory region. In addition, we inserted four tandem repeats of the entire 4-kb upstream fragment, including both conserved regions, into the same reporter vector. These constructs were each injected into the pronucleus of mouse zygotes to create transgenic mice, and expression was assessed using whole-mount LacZ staining of the transgenic embryos at embryonic day 14.5 (E14.5).

Transgenic mice harboring the whole 4-kb reporter showed some staining of LacZ in joints (Fig. 1A). However, LacZ was also detected in the interphalangeal spaces and other tissues. This indicates that the whole 4-kb region contains a segment related to Wnt9a expression in joints but is not specific to joints. Mice carrying a reporter for the 170-bp region just upstream of the transcriptional start site showed no LacZ staining in joints, although they did show expression elsewhere, including in the perichondrium (Fig. 1A). On the other hand, embryos carrying the reporter for the 240-bp region located $4 \mathrm{~kb}$ upstream of the transcription start site showed strong LacZ expression in joints $(n=7 / 7$, LacZpositive transgenics assessed) (Fig. 1A; Supplemental Fig. S2). Although LacZ expression was also observed in the face, in the context of the limbs, its expression appeared to be specific to the joints.
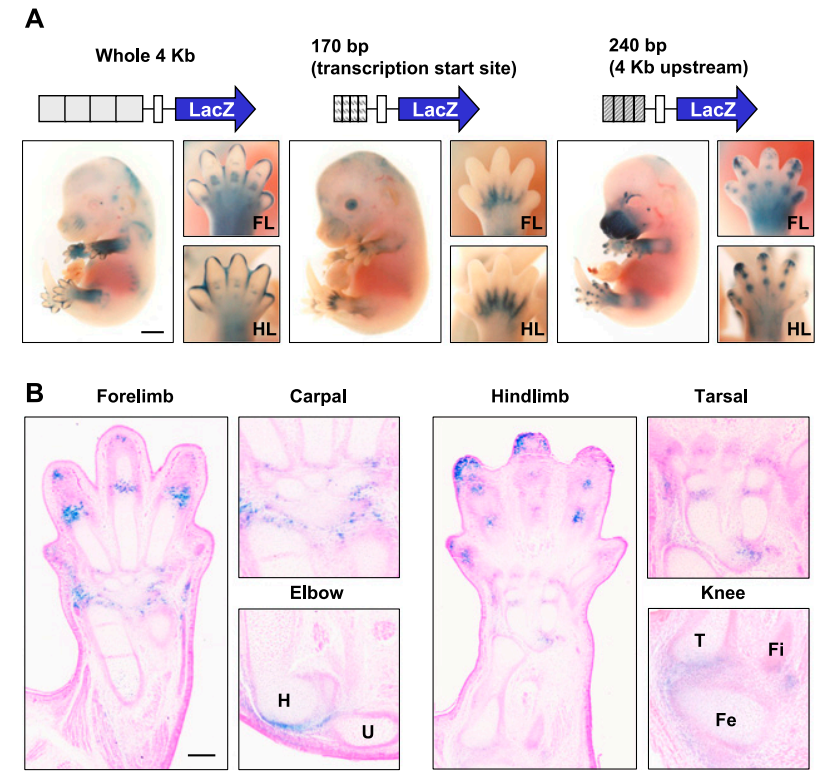

Figure 1. Identification of joint-specific enhancer in the Wnt9a promoter. (A) Whole-mount lacZ stainings of E14.5 transgenic mice embryos harboring four tandem repeats of putative regulatory sequences cloned upstream of an Hsp68 minimal promoter and a LacZ reporter. Three transgene constructs were examined: a 4-kb fragment including both conserved regions as well as the separate 170- and 240-bp regions (see detailed information in Supplemental Figs. S1, S2). Forelimbs (top right) and hindlimbs (bottom right) are magnified. Bar, $1 \mathrm{~mm}$. (B) Paraffin sections of the transgenic embryo harboring the reporter construct carrying the 240-bp region. (H) Humerus; (U) ulna; (Fe) femur; (T) tibia; (Fi) fibula. Bar, $200 \mu \mathrm{m}$.

To define the location of the staining more precisely, we prepared paraffin sections of LacZ-stained embryos carrying the 240-bp construct. Examination of these sections confirmed that LacZ was specifically expressed in the phalangeal, carpal, elbow, tarsal, and knee joints (Fig. 1B). These data suggest that the 240-bp region likely contains regulatory sequences directing Wnt9a expression in joints.

\section{c-Jun expression suggests a role in regulating joint-specific expression of Wnt9a}

To identify candidate transcription factors binding to the identified Wnt9a joint-regulating sequences, we used the TFsearch program (http://www.cbrc.jp/ research/db/ TFSEARCH.html) to analyze the 240-bp sequence in silico. This program screens for consensus binding sites for known transcription factors within a sequence of interest. This program generated a map of 16 putative transcription factor-binding sites, representing potential target sequences for nine different factors (Gata1/2, Cebpb, Pou3f2, AP1, Mzf1, Prx2, Nkx2.5, and p300) within the conserved element (Supplemental Fig. S3). We analyzed the expression patterns of each of these nine factors-or family of factors in the case of Gata1/2, AP1, and Prx2-in the developing mouse limb by whole-mount in situ hybridization of mouse embryos at E12.5 and section 
in situ hybridization at E14.5. Several of the genes analyzed were expressed in developing joints, including c-Jun, JunB, JunD, p300, and Pou3f2 (Fig. 2A,B; Supplemental Fig. S4). Among them, $c$-Jun stood out as being specifically and strongly expressed at developing joints. Further suggesting that this gene might be important in regulating $W n t 9 a$ in joints, we found that its joint-specific expression is conserved in chicks (Supplemental Fig. S5). Section in situ hybridization of mouse embryos from E12.5 to E14.5 revealed that c-Jun is already expressed in the prospective joint region by E12.5 and that its expression grows stronger in joints over time in a pattern very similar to that previously reported for $G d f 5$ and Wnt9a (Fig. 2B). Coexpression of c-Jun and Wnt9a was confirmed by double fluorescence in situ hybridization to hindlimbs at E14.5 (Fig. 2C). In addition, c-Jun protein in developing joints was found to be phosphorylated at Ser 63 or Ser 73 residues by immunohistochemistry, indicating that c-Jun is in an activated form in the developing joints (Fig. 2D).

\section{Demonstration of direct c-Jun binding to a site in the Wnt9a promoter}

To examine the ability of $c$-Jun to induce endogenous Wnt9a expression, we created C3H10T1/2 cells stably expressing $c$-Jun through retroviral transfection. An increased level of $c$-Jun in the cells was confirmed by RTPCR and Western blotting (Supplemental Fig. S6). Overexpression of $c$-Jun enhanced endogenous Wnt9a expression, whereas that of a truncated form of $c$-Jun, which lacks its $\mathrm{N}$-terminal transactivation domain (c-Jun $\Delta N$, also known as Tam67), suppressed the Wnt9a expression (Fig. 3A; Brown et al. 1993).

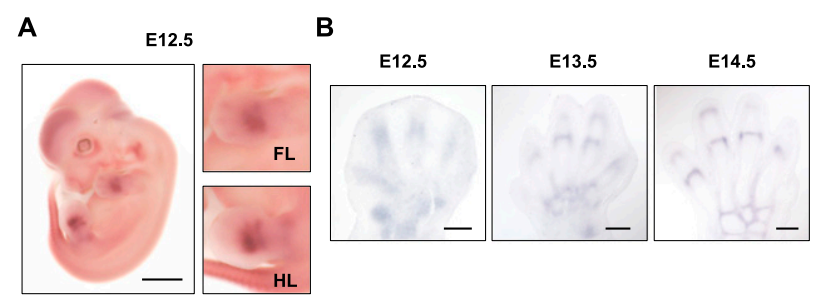

C

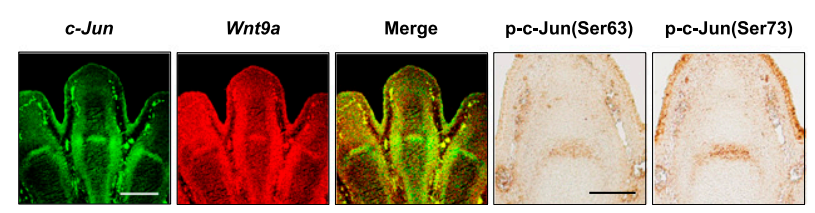

Figure 2. c-Iun is specifically expressed in developing joints. (A) Whole-mount in situ hybridization with antisense probes for c-Jun in mouse embryos (E12.5). A forelimb (FL) and hindlimb (HL) are magnified. Bar, $1 \mathrm{~mm}$. (B) Time course (E12.5-E14.5) of the expression patterns of $c$-Jun in hindlimbs by section in situ hybridization. Bars, $200 \mu \mathrm{m}$. (C) Coexpression of c-Jun and Wnt9a in E14.5 hindlimbs by double fluorescence in situ hybridization. Bar, $200 \mu \mathrm{m}$. (D) Immunohistochemistry of phosphorylated c-Jun at Ser 63 and Ser 73 residues in E14.5 hindlimbs. Bar, $100 \mu \mathrm{m}$.

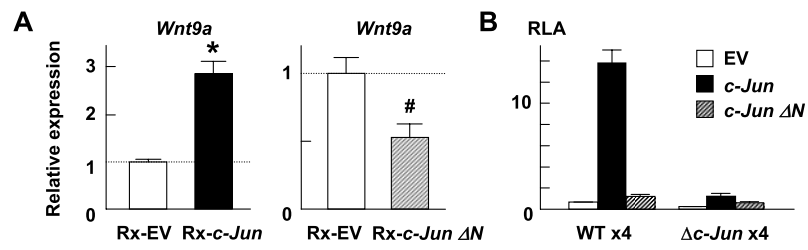

C

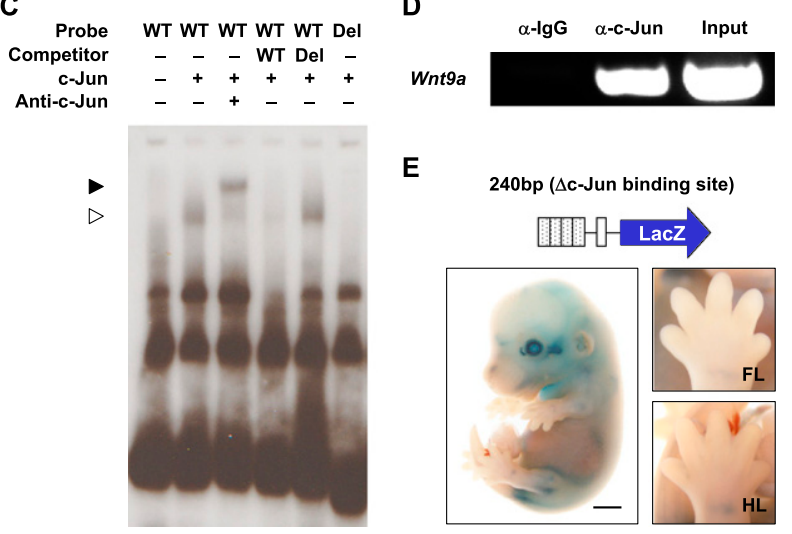

Figure 3. c-Jun regulates $W n t 9 a$ expression through the putative 240-bp enhancer. (A) Wnt9a mRNA levels in C3H10T1/2 cells retrovirally transfected with the control vector (Rx-EV), $c$-Jun ( $\mathrm{Rx}$-c-Jun), or a truncated form of $c$-Jun $(\mathrm{Rx}$-c-Jun $\Delta N)$. The mRNA levels were determined by RT-PCR and are expressed as means (bars) \pm SDs (error bars) for four replicates per construct. $\left.{ }^{\star}{ }^{\star}\right) P<0.001$; (\#) $P<0.01$. (B) Luciferase assay of the transcriptional activity of the transgene driven by four tandem repeats of the wild-type 240-bp enhancer (WT $\times 4$ ) or a similar construct lacking the putative $c$-Jun-binding site $(\Delta c$-Jun $\times 4)$ in C3H10T1/ 2 cells. Empty vector (EV), c-Jun, or c-Jun $\Delta N$ was used as an effecter gene. Relative luciferase activity (RLA) levels are shown as means (bars) \pm SDs (error bars) for duplicates per construct. (C) EMSA for binding between c-Jun protein and the Wnt9a promoter. The wild-type (WT) probe or that lacking a putative c-Jun-binding site (Del) was used. An open arrowhead indicates the shifted band of the c-Jun-DNA probe complex, and a solid arrowhead indicates the band supershifted by an antibody to c-Jun. Cold competitions with a 50 -fold excess of the unlabeled probes are shown. $(D)$ ChIP assay with E14.5 mouse limbs for in vivo binding of c-Jun and its putative binding site in the Wnt9a promoter. (E) Whole-mount lacZ staining of E14.5 transgenic mice carrying a construct encoding four tandem repeats of the 240-bp enhancer lacking the putative c-Jun-binding site. Bar, $1 \mathrm{~mm}$.

The TFsearch program identified a putative TRE element, a site potentially binding c-Jun protein (aaTGACTCAtg, capital letters refer to the putative core-binding site), within the 240-bp sequence (Supplemental Fig. S3). We next used an in vitro assay to determine whether this potential binding site is indeed sufficient to convey c-Jun regulation on associated transcription units. Transcriptional activity of a luciferase reporter construct containing one to four tandem repeats of the 240-bp enhancer was up-regulated by c-Jun overexpression, dependent on the copy number of the enhancer (Supplemental Fig. S7). The reporter transcription was abrogated by the deletion of the c-Jun putative binding site from the 240-bp enhancer or in the case of expressing the truncated c-Jun 
variant lacking the $\mathrm{N}$ terminus domain (Fig. 3B; Supplemental Fig. S7). These results are consistent with c-Jun transactivating the endogenous Wnt9 a promoter through this putative binding site.

We further tested the binding between c-Jun protein and the Wnt9a promoter using an electromobility shift assay (EMSA). We prepared a 51-bp-length digoxigenin (DIG)-labeled oligonucleotide probe including the putative c-Jun-binding site (Supplemental Fig. S3). EMSA showed complex formation between the oligonucleotide probe and in vitro translated c-Jun protein, which was supershifted by an antibody to c-Jun (Fig. 3C). Cold competition with a 50-fold excess of the unlabeled wildtype probe suppressed the complex formation, whereas unlabeled probe lacking the c-Jun-binding site did not affect the complex formation. Moreover, the probe lacking the c-Jun-binding site no longer bound the protein (Fig. 3C). To verify that these results reflect interactions occurring in vivo, a chromatin immunoprecipitation (ChIP) assay was performed using wild-type E14.5 mouse limbs. Cell lysates of E14.5 limbs were amplified with a primer set $(-4015 /-3527)$ spanning the $240-\mathrm{bp}$ enhancer. Indeed, antibodies to c-Jun pulled down the conserved 240-bp sequence upstream of the Wnt9a gene, indicating in vivo binding of c-Jun to the Wnt $9 a$ enhancer (Fig. 3D).

To know the relevance of c-Jun binding to the Wnt $9 a$ enhancer in vivo, we created transiently transgenic mice carrying a construct encoding four tandem repeats of the 240-bp enhancer lacking the putative c-Jun-binding site. Compared with transgenic mice harboring the wild-type enhancer, the LacZ staining in developing joints was very faint (cf. Figs. 3E and 1A), indicating that c-Jun is a major regulator of the Wnt9a expression in joints.

\section{c-Jun deletion in early mesenchyme causes cartilaginous continuity between juxtaposed skeletal elements}

The results described above suggest that $c$-Jun might play a role in establishing joints, through regulation of Wnt9a and potentially other genes. To examine this possibility, we wanted to remove $c$-Jun function from limbs prior to joint formation. Since mouse embryos carrying null mutations in c-Jun die prior to limb development (Hilberg et al. 1993; Eferl et al. 1999), we took advantage of a preexisting c-Jun conditional allele in conjunction with the Prx1-cre transgene that targets the mesenchyme in the early developing limb (Logan et al. 2002).

Exclusive of the joints, skeletal development of long bones in Prx1-cre; c-Jun FL/FL mice was normal at postnatal day 0 (P0) (Supplemental Fig. S8). However, joint formation is disrupted from early stages. The interzone, characterized by condensed localized cells, is the first observable morphological change during the process of joint formation. The interzone is formed at E14.5 in interphalangeal joints of control mice, while no such cell aggregates are visible in c-Jun mutants (Fig. 4A). The efficient cre recombination was confirmed by immunohistochemistry for c-Jun and phosphorylated c-Jun (Supplemental Fig. S9). By E16.5 in wild-type mice, there is

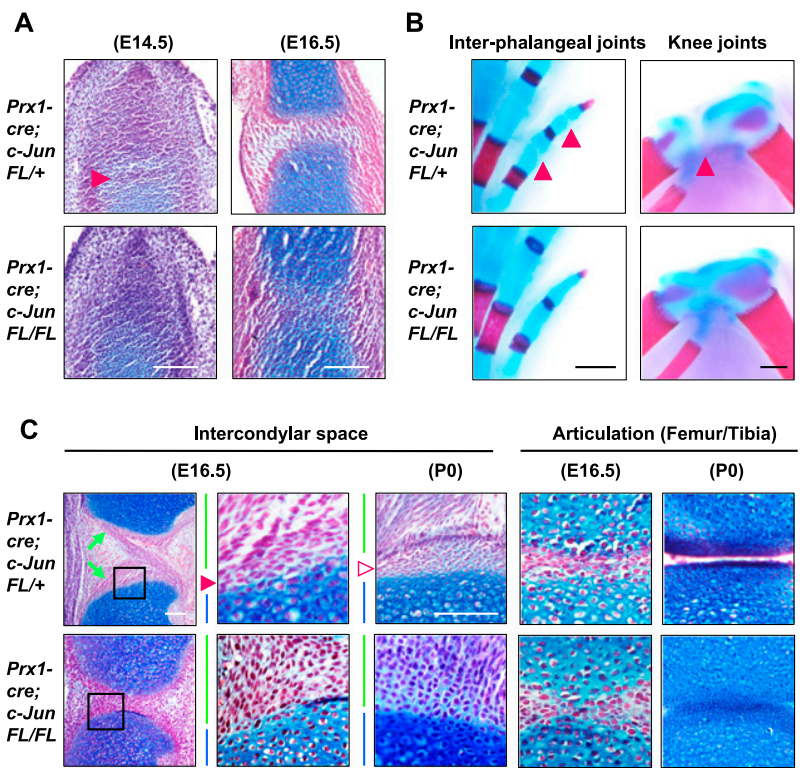

Figure 4. Deletion of c-Jun from early mesenchyme causes cartilaginous continuity between juxtaposed skeletal elements. (A) Alcian blue staining on sections of interphalangeal joints in c-Jun mutants and control mice (E14.5 and E16.5). A red arrowhead indicates a joint interzone, characterized by condensed localized cells in control mice. Bars, $100 \mu \mathrm{m}$. (B) Skeletal staining of interphalangeal and knee joints of $c$-fun mutants and control mice at P0. Red arrowheads indicate the absence of Alcian blue staining at joint spaces in control mice. Bars, $500 \mu \mathrm{m}$. (C) Alcian blue staining on sections of the knee joints of $c$-Jun mutants and control mice at E16.5 and P0. Intercondylar space and articulation between femoral condyle and tibial plateau are shown separately. Green arrows show cruciate ligaments in control mice, which are not visible in c-Jun mutants. Boxed areas are magnified on the right. Green lines on the side indicate a joint cavity space where ligaments normally arise. A solid red arrowhead shows articular cartilage layer at E16.5, characterized by condensed cell layers with a decrease in Alcian blue staining. The layer is more evident at P0 (open red arrowhead). A blue line indicates cartilage condensation in the tibia. Bars, $100 \mu \mathrm{m}$.

a clearly visible joint space in the interphalangeal joints; however, these joint spaces are not well formed in the mutants (Fig. 4A; Supplemental Fig. S10). By P0, stains of Alcian blue, which highlights the cartilage matrix, shows that the normal spans between the phalanges are replaced by relatively uniform staining tissue (Fig. 4B). Similarly, the normal spaces in Alcian blue staining are absent in the developing knee joint at P0 (Fig. 4B). These joint abnormalities were also observed in the radiocarpal joints and interphalangeal joints of forelimbs (Supplemental Fig. S11). Importantly, however, these cartilaginous bridges do not abrogate range of joint motion completely, as the affected joints can be mobilized manually at PO.

To investigate the abnormalities of these joints in more detail, we prepared paraffin sections of E16.5 and P0 knee joints and stained them with Alcian blue. In control mice, cruciate ligaments are already well differentiated in an intercondylar space of the knee at E16.5 (Fig. 4C, green arrows); however, they could not be seen in the mutants. 
In addition, in control mice, there is a distinct articular cartilage layer distal to the chondrocytes of the skeletal condensation (Fig. 4C, solid red arrowhead). This articular cartilage layer is distinguishable by densely packed cells with a flattened shape. No such articular layer can be observed in c-Jun mutants. The distinct morphology of the articular cartilage layer between control mice and c-Jun mutants is even more evident at PO (Fig. 4C, open red arrowhead). Similarly, the articulation between the femur and tibia shows cartilaginous continuity in c-Jun mutants (Fig. 4C).

\section{Interzone formation is disrupted in c-Jun mutants}

We next used molecular markers to investigate the steps of joint formation that are affected in c-Jun mutants. The earliest event in joint formation involves a fate change of proliferating chondrocytes into interzone cells. This begins with down-regulation of chondrogenic markers such as Sox9, Col9a1, Col2a1, and Aggrecan (Acan). Importantly, all of these genes are down-regulated in a normal manner in the E14.5 interphalangeal joints of c-Jun mutants, indicating that the location of the joints is specified normally (Fig. 5A; Supplemental Fig. S12). As the presumptive interzone cells cease to express chondrocyte-specific genes, they begin to differentiate down a different pathway by implementing an interzone-specific transcriptional program. Some early interzone markers, such as Gdf5, were also found to be expressed in the absence of $c$-Jun activity, albeit at a slightly decreased level (Fig. 5A; Supplemental Fig. S13). However, other early interzone markers, such as Autotaxin and Chordin (Hartmann and Tabin 2001), were undetectable in developing joints of $c$-Jun mutants (Fig. 5A).

A similar pattern of aborted differentiation and abnormal joint function is seen in other joints within the developing limb. Similar to the interdigital joints, there is a cell layer that no longer expresses Colga1 at the articulation between the femur and tibia in both control and mutant E16.5 embryos (Supplemental Fig. S14A). However, a characteristic condensed layer of flattened interzone cells can be histologically observed in control mice, while it is missing and there are only round-shaped cells in c-Jun mutants (Supplemental Fig. S14A).

Since the articular cartilage layer is abnormal at E16.5 and $\mathrm{PO}$ in a $c$-Jun-deficient embryo (Fig. 4C), we next examined expression of $\operatorname{Prg} 4$, a lubricant protein that surfaces the articular cartilage. Prg4 is produced by the articular chondrocytes and synovium (Rhee et al. 2005). Although Prg4 mRNA is expressed in both articular chondrocyte and synovial cells in control mice, its expression is greatly decreased in the mutant mice (Supplemental Fig. S14B). These results indicate that c-Jun is required for proper differentiation of the cell types of the joint.

c-Jun is involved in postnatal joint maturation

Prx1-cre; c-Jun FL/FL mice are viable, allowing us to examine the effect of loss of c-fun activity on mature

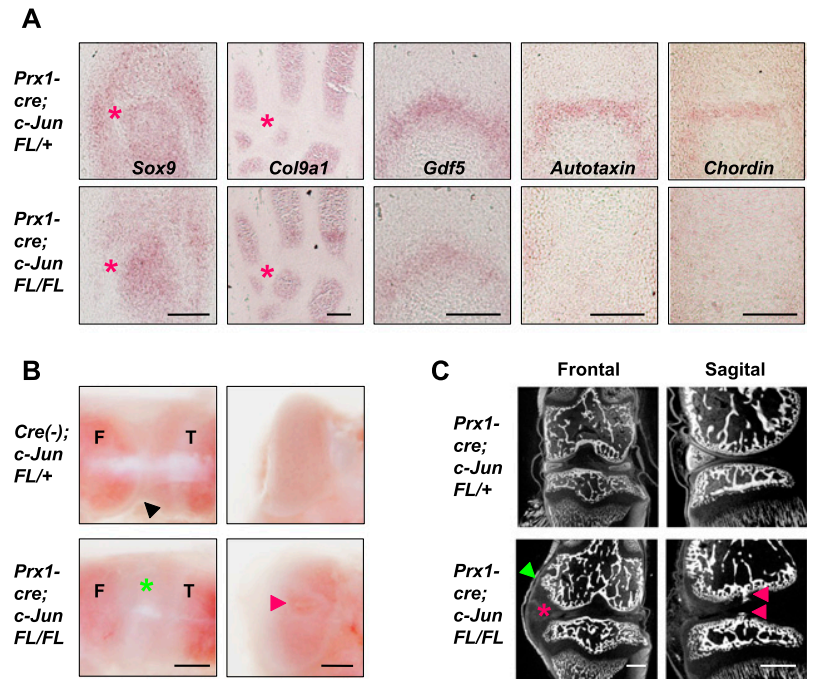

Figure 5. c-Jun plays an essential role in interzone formation and subsequent joint maturation. (A) Section in situ hybridization for chondrogenic and early joint markers Sox9, Col9a1, Gdf5, Autotaxin, or Chordin in interphalangeal joints of c-fun mutants or control mice at E14.5. Red asterisks show the absence of signals between cartilage condensations. Bars, $100 \mu \mathrm{m}$. (B) Macroscopic view of medial femoro-tibial joints at $4 \mathrm{wk}$ old. A solid black arrowhead indicates a transparent layer of well-differentiated articular cartilage in control mice. A green asterisk indicates an abnormal cartilaginous white tissue bridging the femur and tibia in c-Jun mutants. A hollow in the femoral articular surface is shown by a red arrowhead. (F) Femur; (T) tibia. Bars, $500 \mu \mathrm{m}$. (C) MicroCT images of $c$-Jun mutant knee joints at 4 wk old. Frontal and sagital images are shown. A red asterisk indicates an abnormal cartilage tissue observed in $B$. A green arrowhead shows a bulky osteophyte in a femoral condyle. Irregular joint surfaces in c-Jun mutants are indicated by red arrowheads. Bars, $500 \mu \mathrm{m}$.

joints. In control 4-wk-old mice, articular cartilage of the knee is a transparent and smooth layer on the femoral condyle (Fig. 5B, solid arrowhead). However, in the mutant mice, the articular surfaces are not smooth, and a hollow can be observed in the center of each femoral condyle (Fig. 5B, red arrow). Moreover, white cartilaginous tissues bridge between the tibia and femur (Fig. 5B, green asterisk), and collateral ligaments are thinner in c-Jun mutants. Abnormal joint formation can similarly be observed in hip joints. The femoral heads in c-Jun mutants are hypoplastic, and their ligamentum teres are also missing (Supplemental Fig. S15). All of these joint abnormalities were consistently observed in the mutant mice $(n=10 / 10)$.

To further confirm the difference in joint morphology between postnatal control and mutant mice, we performed microCT analysis of their knee joints. Consistent with the macroscopic observations, irregular articular surfaces with bony spurs and ectopic cartilaginous tissue bridging femur and tibia were confirmed in the mutants (Fig. 5C; Supplemental Movies 1, 2). In addition, the patellar ligament and meniscus were underdeveloped in the mutants. 


\section{Positive feedback regulation of c-Jun in joints}

Previous reports have shown that phosphorylated c-Jun protein can interact with the c-Jun promoter and increases its own transcriptional activity in colon cancer (Nateri et al. 2005). This raised the possibility that c-Jun might also participate in a positive feedback loop in joints. To test this, we examined the expression of the c-Jun 5' untranslated region (UTR) $(+63 /+549$, still present following cre-mediated inactivation of the c-Jun locus) using specific probes for in situ hybridization (Supplemental Fig. S16A). In situ hybridization revealed that the mRNA expression of c-Jun 5' UTR in joints is partially suppressed, while that of $c$-Jun coding sequence (CDS) is completely missing in Prx1-cre; c-Jun FL/FL mice (Supplemental Fig. S16B). Quantification with RT-PCR confirmed the partial suppression of $c$-Jun transcription in the mutants (Supplemental Fig. S16C). These results indicate that c-Jun does have a positive feedback loop, enhancing its own expression in the joints that might serve a role in maintaining high expression levels of c-Jun in the joint-specific environment.

\section{Other AP-1 family molecules are not strongly expressed in developing joints}

Although c-Jun can form homodimers, several related AP1 family members are known to form heterodimeric complexes with c-Jun, including c-Fos, FosB, JunB, JunD, Fra1, Fra2, and Atf2 (Halazonetis et al. 1988; Nakabeppu et al. 1988). We also found a potential c-Jun heterodimerbinding sequence, "TGAGCTCA," in the identified 240-bp sequence (Supplemental Fig. S2, first row). To know whether any were potential partners for c-Jun, we analyzed the expression patterns of each of these molecules by section in situ hybridization to E14.5 hindlimbs. JunB, JunD, Fra2, and Atf2 are weakly expressed in developing joints and were also found at a comparable level in the surrounding perichondrium, while other AP1 family molecules (c-Fos, FosB, and Fra1) were not detectable in developing joints (Supplemental Figs. S3B, S17). Moreover, none of these AP-1 family members were upregulated in the joints of $c$-Jun mutants (Supplemental Fig. S17). Thus, of the family members investigated, only JunB, JunD, Fra2, and Atf2 are potential heterodimer partners, and none are fully able to compensate for loss of c-Jun activity.

\section{c-Jun is necessary for both Wnt9a and Wnt16 expression}

Promoter analysis identified $c$-Jun as a potential upstream regulator of Wnt9a. Consistent with this, fluorescence in situ hybridization shows that Wnt9 a expression is lost, at the level of detection, in the joints of $c$-Jun mutants at E14.5 (Fig. 6A). However, c-Jun mutants exhibit a much severer phenotype than that reported for Wnt9a-deficient mice (Spater et al. 2006b). This raises the possibility that c-Jun might regulate expression of other molecules acting in an at least partially redundant manner with Wnt9a, such as other Wnt family genes. Wnt4 and Wnt16 are

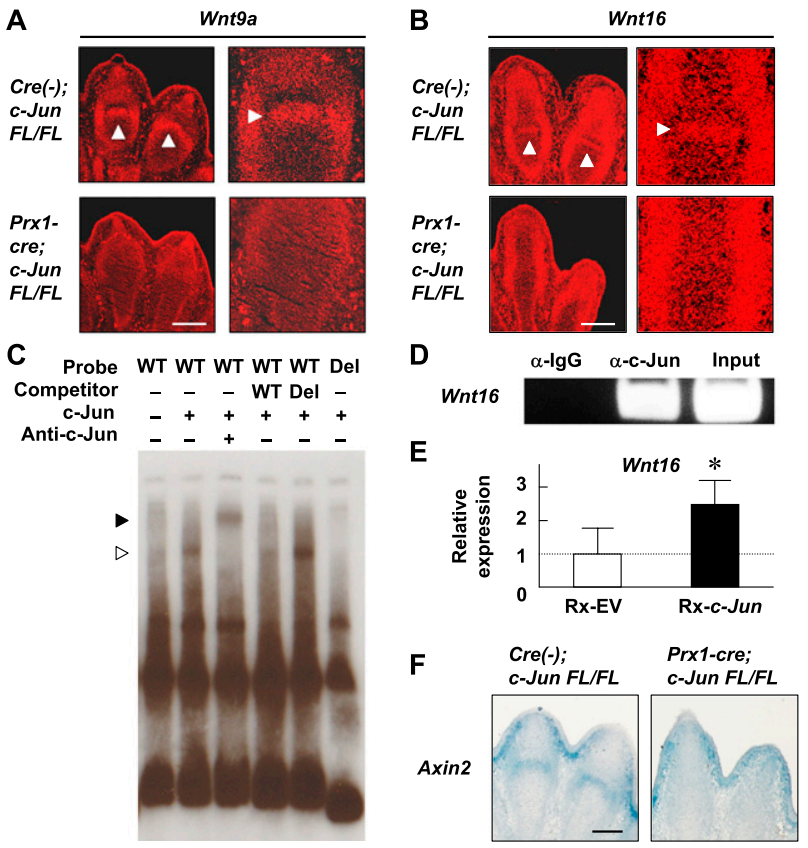

Figure 6. c-Jun enhances multiple Wnt expressions in joints. (A) Fluorescence in situ hybridization of Wnt9a on a section of E14.5 hindlimb. White arrowheads show positive signals in interphalangeal joints of control mice. The joints are magnified on the right. Bar, $200 \mu \mathrm{m}$. (B) Fluorescence in situ hybridization of Wnt16 on a section of E14.5 hindlimb. Bar, $200 \mu \mathrm{m}$. (C) EMSA for binding between c-Jun protein and the Wnt16 promoter. The wild-type (WT) probe or that lacking a putative c-Jun-binding site (Del) in the Wnt16 promoter was used. An open arrowhead indicates a shifted band of the c-Jun-DNA probe complex, and a solid arrowhead indicates the band supershifted by an antibody to c-Jun. Cold competitions with a 50 -fold excess of the unlabeled probes are shown. $(D)$ ChIP assay with E14.5 limbs for showing in vivo binding of $c$-Jun and its putative binding site in the Wnt16 promoter. $(E)$ Wnt16 mRNA levels in C3H10T1/2 cells retrovirally transfected with the control vector $(\mathrm{Rx}-\mathrm{EV})$ or c-Jun (Rx-c-Jun). The mRNA levels were determined by RT-PCR and are expressed as means (bars) \pm SDs (error bars) for four replicates per construct. $\left(^{\star}\right) P<0.05$. $(F)$ Section in situ hybridization of Axin2 on a section of interphalangeal joints of c-Jun mutants at E14.5. Bar, $200 \mu \mathrm{m}$.

known to be expressed in mouse joints in addition to Wnt9a (Guo et al. 2004), and we confirmed that the expression of these Wnt family members is phylogenetically conserved in chicks (Supplemental Fig. S5). We therefore checked their expression in c-Jun mutants and wild-type mice by in situ hybridization. Wnt4 expression does not appear to be affected in c-Jun mutants (Supplemental Fig. S18). However, Wnt16 expression in the joint interzone was lost in the mutants along with Wnt9a (Fig. 6B).

These data suggest that $c$-Jun might regulate the Wnt16 promoter in addition to the Wnt9 $a$ promoter. To examine a potential direct interaction between c-Jun and sequences regulating the Wnt16 gene, we next looked for putative $c$-Jun-binding sites in the $5^{\prime}$ region of the Wnt16 transcriptional start site. We indeed identified a fairly well-conserved c-Jun-binding site at $3.5 \mathrm{~kb}$ upstream of 
the Wnt16 transcription start site $(-3545 /-3539)$ (Supplemental Fig. S19). EMSA showed direct binding between c-Jun and this predicted site (Fig. 6C). ChIP assay with cell lysates of the E14.5 limb also confirmed that the putative binding site upstream of Wnt16 could be pulled down by an anti-c-Jun antibody (Fig. 6D). Moreover, retroviral overexpression of $c$-Jun in C3H10T1/2 cell lines significantly enhanced endogenous expression of Wnt16 (Fig. 6E).

Intracellular signaling downstream from $W n t 9 a$ and Wnt16 are mediated by the canonical Wnt- $\beta$-catenin pathway, whereas Wnt4 can act through either the canonical or noncanonical pathway, depending on context (Guo et al. 2004; Sugimura and Li 2010). To assess $\beta$-catenin signaling in the joints of $c$-Jun mutants, we examined the expression of Axin2, which is a classical canonical Wnt target gene (Jho et al. 2002). Axin2 is expressed in developing joints as well as epidermis in control mice, while the expression in joints, but not in the epidermis, is completely suppressed in c-Jun mutants (Fig. 6F). Similarly, we analyzed the localization of $\beta$-catenin by immunohistochemistry and found that $\beta$-catenin resides in the nucleus of many interzone cells in control mice; however, it is largely in the cell membrane in c-Jun mutants (Supplemental Fig. S20). These results demonstrate that $c$-Jun regulates $\mathrm{Wnt} / \beta$-catenin activities in joints and, taking into account our Wnt gene analysis, likely does so by enhancing Wnt9a and Wnt16 expression.

\section{Discussion}

Unlike other aspects of skeletogenesis, the transcriptional regulation of joint formation has not been established. We identified the transcriptional factor $c$-Jun as a regulator of Wnt9a during joint formation. c-Jun binds to the Wnt9a enhancer and is necessary for Wnt9a transcription in vivo. The loss of $c$-Jun activity produces a stronger joint phenotype than loss of Wnt9a, suggesting that it regulates additional targets during joint formation. We identified Wnt16 as one of these additional targets, likely acting in a partially redundant role with $W n t 9 a$. In addition to maintaining Wnt canonical activity necessary for the early development of joints, we found that c-Jun is also required for joint maturation in later stages.

\section{c-Jun and the canonical Wnt signaling pathway} in joints

Several Wnt molecules, including Wnt4, Wnt9a, and Wnt16, are expressed in early joint progenitor cells and hence potentially could play redundant roles in joint formation (Guo et al. 2004). Wnt proteins signal through at least two distinct pathways: the $\beta$-catenin-mediated canonical pathway and the noncanonical pathway. Wnt4 has been implicated in both the canonical and noncanonical pathways (Guo et al. 2004; Sugimura and Li 2010); however, Wnt9 $a$ and Wnt16 are considered to be mediated only by the canonical pathway (Guo et al. 2004). Many lines of evidence suggest that the canonical path- way is important during joint development (Guo et al. 2004; Spater et al. 2006a; Kahn et al. 2009). We found a dramatically decreased level of Axin2, a classical Wnt canonical target, in the developing joints of c-Jun mutants. In addition, we also showed that the translocation of $\beta$-catenin into the nucleus in developing joints does not occur in c-Jun mutants. As Wnt9a and Wnt16 expression are both lost in the absence of $c$-Jun, but Wnt4 expression is not affected, it suggests that Wnt4 acts through a nonredundant, noncanonical pathway in this context.

Interestingly, c-Jun has also been described as acting in the noncanonical planar cell polarity pathway. However, interactions between canonical and noncanonical pathways have recently been reported, and c-Jun has been implicated as an important regulator of the $\beta$-cateninmediated canonical pathway in addition to its role in the noncanonical pathway (Nateri et al. 2005; Gan et al. 2008; Saadeddin et al. 2009). In particular, c-Jun protein forms a complex with nuclear Dvl, $\beta$-catenin, and TCF, which leads to stabilization of the $\beta$-catenin-TCF interaction (Gan et al. 2008). It is possible that this interactive function of $c$-Jun might be an additional reason why canonical Wnt activities are severely suppressed in developing joints of c-Jun mutants in addition to the decreased level of Wnt ligands Wnt9 $a$ and Wnt16.

\section{c-Jun is the only AP1 family member likely to play a pivotal role in joint formation}

Among AP1 family genes, JunB, JunD, Fra2, and Atf2 are the only genes other than $c$-Iun that are expressed in developing joints at a level detected by our section in situ hybridization analysis. However, their expression levels are modest in the joint interzone, and mice deficient in these genes do not exhibit joint abnormalities. JunB-deficient mice show osteopenia (Kenner et al. 2004), while JunD-deficient mice exhibit increased bone mass (Kawamata et al. 2008). Fra2-deficient mice and Atf2-deficient mice show abnormal chondrogenic differentiation in long bones during the process of endochondral ossification (Reimold et al. 1996; Karreth et al. 2004). On the other hand, c-Jun deficiency in this study results in striking joint abnormalities throughout the limbs. These facts strongly indicate that c-Jun is the most important AP1 family member for joint formation. Nevertheless, it remains possible that $\operatorname{JunB}$, JunD, Fra2, and/or Atf2 form heterodimers with $c$-Jun during normal joint development and that each can compensate for the others in the process.

We showed that c-Jun is phosphorylated in developing joints by immnohistochemical analysis, indicating that it exists in an activated form in developing joints. However, JunAA phospho-dead mutants, where the endogenous c-Jun gene is replaced by a mutant c-Jun allele with Ser 63 and Ser 73 mutated to alanines, are reported to be viable and healthy (Behrens et al. 1999). Taken together with the phenotype that we observed in the absence of c-Jun, this indicated that there must be other mechanisms to activate c-Jun besides phosphorylation of these two serine residues. 
Independent roles of c-Jun and Gdf5 in interzone formation

In addition to Wnt9a, a second secreted protein, Gdf5, is expressed in the early developing joint (Storm and Kingsley 1999). We found that Gdf5 expression is partially suppressed in c-Jun mutants, albeit to a small extent. Although it is thus clear that Gdf5 is mainly regulated by mechanisms that are independent of $c$-Jun, the partial reduction in $G d f 5$ activity that we observed is consistent with the finding that Wnt9a misexpression induces ectopic Gdf5 expression in chicks (Hartmann and Tabin 2001).

\section{c-Jun is required early in joint development}

c-Jun is expressed in early stage developing joints. We produced $c$-Jun conditional- deficient mice using the Prx1cre transgene, which targets the mesenchyme in the early developing limb. These mice exhibit severe joint phenotypes from the earliest step of interzone formation, which is the first morphological change observable during joint development. In contrast, mice in which c-Jun activity is removed from chondrocytes at a later stage using a cre allele under the human COL2A1 promoter have morphologically normal limb skeletal elements, although they show malformed, scoliotic vertebral columns and abnormal morphogenesis of the rib cages (Behrens et al. 2003). Thus, once mesenchymal cells decide to adopt a cartilage cell fate, c-Jun no longer appears to be relevant for their subsequent development.

\section{The process of joint formation}

Broad steps of joint formation, including interzone formation, cavitation, and subsequent differentiation, are severely affected in c-Jun mutants. Based on our results, we can place $c$-Jun into the cascade-establishing joints, as follows (Fig. 7A-D). The first key event in joint formation involves diverting the differentiation pathway of the prechondrogenic condensation from chondrocyte to interzone through an unknown mechanism. Prospective joint cells in the prechondrogenic condensation lose their chondrogenic characteristics in the specific prospective joint sites, characterized by the decrease of Sox9, Col9a1, Col2a1, and Acan expressions (Fig. 7B). c-Jun mutants show normal suppression of chondrogenic marker genes in the prospective joint sites. c-Jun is thus not involved in this initial process defining the location of the joints.

c-Jun does appear to play an important role in completing differentiation into interzone cells and subsequent maturation of developing joints. c-Jun mutants show the abnormal formation of joint interzone molecularly and morphologically, including failure to express several interzone-specific genes, such as Autotaxin and Chordin (Fig. 7C), and a loss of the characteristically flattened and condensed morphology of the interzone cells. The abnormal round-shaped cells of the mutant interzone are eventually replaced by continuous cartilage tissues bridging two adjacent cartilage condensations. Previous studies of mutants affecting joint formation have described this phenotype as "joint fusions." This is actually a mis-

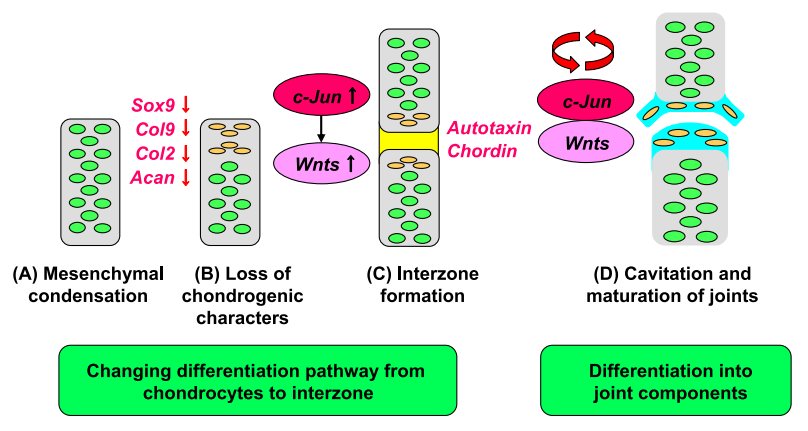

Figure 7. $(A-D)$ Possible model of joint formation in developing limbs. Joint initiation occurs at the specific locations by diverting the chondrogenic differentiation pathway to a pathway leading to the formation of the interzone, which is followed by differentiation into a variety of joint components. (A) Joint initiation arises at a specific location within the mesenchymal condensations. $(B)$ The loss of chondrogenic characters is demonstrated by a decreased level of $\operatorname{Sox} 9, \operatorname{Col} 9$, Col2, and Acan at the prospective joint sites. (C) c-Jun is essential for activating canonical Wnt activities during interzone formation by inducing expressions of Wnt9a and Wnt16. The expression of early interzone marker genes such as Autotaxin and Chordin is up-regulated in response to these signals. (D) c-Jun also contributes to maintaining the jointspecific environment during subsequent cavitation and the maturation of joints.

nomer, as "fusion" implies that two distinct elements grow until they are joined together. Rather, it is clear from our analysis that the joint space never forms in the first place, and the cartilage bridge is a result of a failure of differentiation.

At later stages, the differentiated interzone cells normally give rise to various joint components such as ligaments, articular cartilage, etc. (Fig. 7D). In c-Jun mutants, a range of joint components are malformed or hypoplastic. We identified Wnt9a and Wnt16 as targets of $c$-Jun. Based on the phenotype of Wnt9a mutants, which is similar to the c-Jun loss of function, albeit weaker, we proposed that the decreased level of Wnt canonical activities is a major reason for joint abnormalities in c-Jun mutants. However, it is possible that there are other key downstream targets of c-Jun in developing joints that also play critical roles in this process.

Here we show that c-Jun plays a pivotal role in the formation of the joint interzone, cavitation, and the subsequent differentiation of joint components. c-Jun is a major regulator of $\mathrm{Wnt} / \beta$-catenin canonical activities in joints, and proper levels of $W n t$ activity are critical for joint formation. Further studies will be required to clarify the complex molecular network underlying joint initiation, formation, and maintenance. In particular, we started with Wnt9 $a$ as one of the earliest known markers of joint formation and identified one of its critical upstream regulators, $c$-Jun. As joint initiation still occurs in the proper location in the absence of $c$-Jun, the factors establishing the location of the joints remain to be elucidated. It is possible that these might be found by examining the $c$-Jun promoter to identify factors acting further upstream. 


\section{Materials and methods}

\section{Creating transiently transgenic mice}

All animal experiments were performed following protocols approved by the Harvard Medical School Institutional Animal Care and Use Committee (IACUC). We created constructs with the conserved candidate regions of the mouse Wnt $9 a$ enhancer placed upstream of an Hsp68 minimal promoter and a LacZ reporter gene. Sequences $5^{\prime}$ of the Wnt9 $a$ gene (NC_000077) were amplified by PCR. To enhance reporter sensitivity, we cloned four tandem repeats of each candidate enhancer. For this purpose, four additional sets of restriction enzyme sites (BglII/NheI, NheI/AgeI, AgeI/SbfI, and SbfI/SwaI) were inserted into the multicloning site. After removing the unnecessary sequences, such as the ampicillin-resistant gene, using BglII/SalI, the $4 \times$ enhancer-Hsp68-LacZ cassette was used for microinjection, and injected mouse eggs were transplanted into uteri of pseudopregnant mice. Wholemount LacZ staining of the transgenic embryos was carried out at stage E14.5 by a standard protocol (Kahn et al. 2009) with a small modification: We fixed with $0.2 \%$ glutalaldehyde solutions instead of $4 \%$ paraformaldehyde. After the staining, paraffin sections were prepared, and $1 \%$ eosin was used for counterstaining. Primer information is available in Supplemental Table S1.

\section{Histological analyses of mouse embryos}

For in situ hybridization, partial sequences of each gene of interest were amplified and linked to a $\mathrm{T} 7$ promoter sequence by PCR. DIG-labeled antisense riboprobes were prepared with T7 RNA polymerase using a DIG RNA-labeling kit SP6/T7 (Roche). The mouse embryos were prepared as previously described (McGlinn and Mansfield 2011) and treated with an anti-DIGcoupled alkaline phosphatase (AP). For detection of Axin2 mRNA, the samples were incubated with anti-DIG-streptavidin (Roche), and signals were amplified by a TSA Plus Biotin kit (PerkinElmer). The samples were then treated with an antistreptavidin-coupled AP (Roche). The probed molecules were detected using NBT/BCIP stock solution (Roche) or BM purple (Roche). For fluorescence in situ hybridization, the binding of the Wnt9a or Wnt16 probe was detected using an anti-DIG antibodycoupled POD (Roche) and TSA plus cyanine 3 system (Perkin Elmer). For double in situ hybridization, the fluorescein-labeled c-Jun probe was prepared using a fluorescein RNA-labeling mix (Roche). The probed c-Jun transcript was detected using an antifluorescein antibody-coupled POD (Roche) and TSA plus fluorescein system (Perkin Elmer). For immunohistochemistry, antigen retrieval was performed with $0.01 \mathrm{M}$ sodium citrate. Primary antibodies for c-Jun (1:50; Abcam, ab32137), phosphoc-Jun (Ser 63) (1:50; Cell Signaling Technology, \#9261), phospho-c-Jun (Ser 73) (1:50; Cell Signaling Technology, \#3270), $\beta$-catenin (1:50; Abcam, ab6302), and phospho- $\beta$-catenin (1:50; Abcam, ab53050) were used as primary antibodies, and nonimmune normal rabbit IgG (1:50; Santa Cruz Biotechnology, sc-2027) was used for a control. The CSA II biotin-free catalyzed amplification system (DakoCytomation) was used for diaminobenzidine (DAB) detection. For fluorescent detection, Alexa fluor 488-conjugated goat anti-rabbit antibody (1:250; Jackson ImmunoResearch Laboratories, 111-545-003) was used as a secondary antibody. Fluorescent images were obtained with either an SP2 (Leica) or LSM780 (Carl Zeiss) inverted confocal microscope (Carl Zeiss).

\section{Plasmid construction}

The mouse c-Jun gene (NM_010591) was PCR-amplified and cloned into pMx-IRES-Bsr retroviral expression plasmid (Morita et al. 2000), pCMV-HA (Clontech), or pCITE-4a(+) (Novagen). The truncated $c$-Jun that lacks the $\mathrm{N}$-terminal domain was created by PCR. One to four tandem repeats of the enhancer were cloned into the pGL4.23 [luc2/minP] vector (Promega) for luciferase assays. Primer information for plasmid construction is available in Supplemental Table S1.

\section{Cell cultures}

C3H10T1/2 cells were maintained in Dulbecco's modified Eagle's medium (DMEM) containing 10\% FBS (Invitrogen) supplemented with L-glutamine $(2 \mathrm{mM})$, penicillin $(100 \mathrm{U} / \mathrm{mL})$, and streptomycin $(100 \mathrm{mg} / \mathrm{mL})$. For retroviral gene transfer, Plat-E cells were plated in $60-\mathrm{mm}$ dishes and transfected with $3 \mu \mathrm{g}$ of pMx-IRES-Bsr vector using FuGene6 (Roche) on the following day (Morita et al. 2000). Forty-eight hours after transfection, the medium was collected and used as the retrovirus supernatant, which was applied to C3H10T1/2 cells. Selection of the retrovirally introduced C3H10T1/2 cells was started after $2 \mathrm{~d}$ in the medium containing $10 \mu \mathrm{g} / \mathrm{mL}$ blasticidin. After $2 \mathrm{wk}$, the surviving cells were collected and assayed.

\section{Western blotting}

Cells were collected with M-PER mammalian protein extraction reagent (Pierce Biotechnology). Anti-c-Jun antibody (1:1000; Abcam, ab32137) and anti- $\beta$-Actin antibody (1:1000; Abcam, ab8227) were used for primary antibodies, and peroxidase-conjugated goat anti-rabbit antibody (1:10000; Jackson ImmunoResearch, 111-035-144) was used for a secondary antibody. Detection was performed using ECL Western blotting detection reagents (GE Healthcare).

\section{RT-PCR analysis}

Total RNAs were isolated with an RNeasy minikit (Qiagen), and each sample was treated by DNase, reverse-transcribed with a QuantiTect reverse transcription kit (Qiagen), and used as a template for the second-step PCR. Power SYBR Green PCR master mix (Applied Biosystems) was used for the second-step PCR, and detection was performed with the 7500 Fast Real-Time PCR system (Applied Biosystems). Primer information is available in Supplemental Table S1. Target genes' mRNA was adjusted with mouse Gapdh as an internal control.

\section{Luciferase assay}

Transfection of C3H10T1/2 cells was performed in duplicate in 24-well plates using FuGene6 with plasmid DNA (200 ng of pGL4.23 [luc2/minP] vector, $100 \mathrm{ng}$ of effector vector, $4 \mathrm{ng}$ of pRL-TK vector [Promega] for internal control per well). Cells were harvested $48 \mathrm{~h}$ after transfection. The luciferase assay was performed with a dual luciferase reporter assay system (Promega) using an LMax luminometer (Molecular Devices). Results were shown as the ratio of firefly luciferase activity to renilla activity.

\section{EMSA}

The mouse c-Jun protein was in vitro translated using the TNT quick-coupled transcription/translation system (Promega) and pCITE-4a(+) vector encoding c-Jun. EMSA was carried out using a DIG gel shift kit second generation (Roche). For competition analyses, a 50-fold excess of unlabeled competitor probe was included in the binding reaction. For the supershift experiments, $1 \mu \mathrm{g}$ of anti-c-Jun antibody (Abcam, ab31419) was added. 


\section{ChIP assay}

For ChIP analysis for tissues, E14.5 mouse limbs were homogenized with TissueLyser (Qiagen). Sheared chromatin was prepared using Covaris E210 (Covaris, Inc), and then $10 \mu \mathrm{g}$ of anti-c-Jun antibody (Abcam, ab31419) or normal rabbit IgG (Santa Cruz Biotechnology, sc-2027) was used for immunoprecipitation.

\section{Skeletal staining}

Whole-mount skeletal staining was performed with Alcian blue and Alizarin red using a standard protocol (Ovchinnikov 2009). Alcian blue staining on section was performed with $1 \%$ Alcian blue followed by nuclear fast red solution for counterstaining.

\section{MicroCT analysis}

Knee joints of 4-wk-old mice were harvested. Their soft tissues were removed as much as possible and fixed with $4 \%$ paraformaldehyde overnight. They were put into $0.3 \%$ phosphotungstic acid in $70 \%$ ethanol. Images and movies were obtained with an XCT-200 scanner (Xradia).

\section{Acknowledgments}

We are grateful to Dr. Axel Behrens for a kind gift of $c$-Jun floxed mice. The backbone plasmid for the reporter construct of transiently transgenic mice was a kind gift from Dr. David M. Kingsley. We appreciate Ms. Karen Cox for taking microCT images. This work was supported by NIH grant number PO1DK056246 to C.J.T.

\section{References}

Basit S, Naqvi SK, Wasif N, Ali G, Ansar M, Ahmad W. 2008. A novel insertion mutation in the cartilage-derived morphogenetic protein-1 (CDMP1) gene underlies Grebe-type chondrodysplasia in a consanguineous Pakistani family. BMC Med Genet 9: 102.

Behrens A, Sibilia M, Wagner EF. 1999. Amino-terminal phosphorylation of c-Jun regulates stress-induced apoptosis and cellular proliferation. Nat Genet 21: 326-329.

Behrens A, Haigh J, Mechta-Grigoriou F, Nagy A, Yaniv M, Wagner EF. 2003. Impaired intervertebral disc formation in the absence of Jun. Development 130: 103-109.

Brown PH, Alani R, Preis LH, Szabo E, Birrer MJ. 1993. Suppression of oncogene-induced transformation by a deletion mutant of c-jun. Oncogene 8: 877-886.

Eferl R, Sibilia M, Hilberg F, Fuchsbichler A, Kufferath I, Guertl B, Zenz R, Wagner EF, Zatloukal K. 1999. Functions of c-Jun in liver and heart development. J Cell Biol 145: 1049-1061.

Gan XQ, Wang JY, Xi Y, Wu ZL, Li YP, Li L. 2008. Nuclear Dvl, c-Jun, $\beta$-catenin, and TCF form a complex leading to stabilization of $\beta$-catenin-TCF interaction. J Cell Biol 180: 10871100.

Guo X, Day TF, Jiang X, Garrett-Beal L, Topol L, Yang Y. 2004. Wnt/ $\beta$-catenin signaling is sufficient and necessary for synovial joint formation. Genes Dev 18: 2404-2417.

Halazonetis TD, Georgopoulos K, Greenberg ME, Leder P. 1988. c-Jun dimerizes with itself and with c-Fos, forming complexes of different DNA binding affinities. Cell 55: 917-924.

Hartmann C, Tabin CJ. 2001. Wnt-14 plays a pivotal role in inducing synovial joint formation in the developing appendicular skeleton. Cell 104: 341-351.

Hilberg F, Aguzzi A, Howells N, Wagner EF. 1993. c-jun is essential for normal mouse development and hepatogenesis. Nature 365: 179-181.
Jho EH, Zhang T, Domon C, Joo CK, Freund JN, Costantini F. 2002. Wnt $/ \beta$-catenin/Tcf signaling induces the transcription of Axin2, a negative regulator of the signaling pathway. Mol Cell Biol 22: 1172-1183.

Kahn J, Shwartz Y, Blitz E, Krief S, Sharir A, Breitel DA, Rattenbach R, Relaix F, Maire P, Rountree RB, et al. 2009. Muscle contraction is necessary to maintain joint progenitor cell fate. Dev Cell 16: 734-743.

Karreth F, Hoebertz A, Scheuch H, Eferl R, Wagner EF. 2004. The AP1 transcription factor Fra2 is required for efficient cartilage development. Development 131: 5717-5725.

Kawamata A, Izu Y, Yokoyama H, Amagasa T, Wagner EF, Nakashima K, Ezura Y, Hayata T, Noda M. 2008. JunD suppresses bone formation and contributes to low bone mass induced by estrogen depletion. I Cell Biochem 103: 1037-1045.

Kenner L, Hoebertz A, Beil FT, Keon N, Karreth F, Eferl R, Scheuch H, Szremska A, Amling M, Schorpp-Kistner M, et al. 2004. Mice lacking JunB are osteopenic due to cellautonomous osteoblast and osteoclast defects. I Cell Biol 164: 613-623.

Logan M, Martin JF, Nagy A, Lobe C, Olson EN, Tabin CJ. 2002. Expression of Cre recombinase in the developing mouse limb bud driven by a Prxl enhancer. Genesis 33: 77-80.

McGlinn E, Mansfield JH. 2011. Detection of gene expression in mouse embryos and tissue sections. Methods Mol Biol 770: 259-292.

Miyamoto Y, Mabuchi A, Shi D, Kubo T, Takatori Y, Saito S, Fujioka M, Sudo A, Uchida A, Yamamoto S, et al. 2007. A functional polymorphism in the $5^{\prime}$ UTR of GDF5 is associated with susceptibility to osteoarthritis. Nat Genet 39: 529533.

Morita S, Kojima T, Kitamura T. 2000. Plat-E: An efficient and stable system for transient packaging of retroviruses. Gene Ther 7: 1063-1066.

Nakabeppu Y, Ryder K, Nathans D. 1988. DNA binding activities of three murine Jun proteins: Stimulation by Fos. Cell 55: 907-915.

Nateri AS, Spencer-Dene B, Behrens A. 2005. Interaction of phosphorylated c-Jun with TCF4 regulates intestinal cancer development. Nature 437: 281-285.

Ovchinnikov, D. 2009. Alcian blue/alizarin red staining of cartilage and bone in mouse. Cold Spring Harb Protoc :doi: $10.1101 /$ pdb.prot5170.

Reimold AM, Grusby MJ, Kosaras B, Fries JW, Mori R, Maniwa S, Clauss IM, Collins T, Sidman RL, Glimcher MJ, et al. 1996. Chondrodysplasia and neurological abnormalities in ATF-2deficient mice. Nature 379: 262-265.

Rhee DK, Marcelino J, Baker M, Gong Y, Smits P, Lefebvre V, Jay GD, Stewart M, Wang H, Warman ML, et al. 2005. The secreted glycoprotein lubricin protects cartilage surfaces and inhibits synovial cell overgrowth. J Clin Invest 115: 622-631.

Saadeddin A, Babaei-Jadidi R, Spencer-Dene B, Nateri AS. 2009. The links between transcription, $\beta$-catenin/JNK signaling, and carcinogenesis. Mol Cancer Res 7: 1189-1196.

Spater D, Hill TP, Gruber M, Hartmann C. 2006a. Role of canonical Wnt-signalling in joint formation. Eur Cell Mater 12: $71-80$.

Spater D, Hill TP, O'Sullivan RJ, Gruber M, Conner DA, Hartmann C. 2006b. Wnt9a signaling is required for joint integrity and regulation of thh during chondrogenesis. Development 133: 3039-3049.

Storm EE, Kingsley DM. 1996. Joint patterning defects caused by single and double mutations in members of the bone morphogenetic protein (BMP) family. Development 122: 3969-3979. 
Kan and Tabin

Storm EE, Kingsley DM. 1999. GDF5 coordinates bone and joint formation during digit development. Dev Biol 209: 11-27.

Storm EE, Huynh TV, Copeland NG, Jenkins NA, Kingsley DM, Lee SJ. 1994. Limb alterations in brachypodism mice due to mutations in a new member of the TGF $\beta$-superfamily. Nature 368: 639-643.

Sugimura R, Li L. 2010. Noncanonical Wnt signaling in vertebrate development, stem cells, and diseases. Birth Defects Res C Embryo Today 90: 243-256.

Tsumaki N, Nakase T, Miyaji T, Kakiuchi M, Kimura T, Ochi T, Yoshikawa H. 2002. Bone morphogenetic protein signals are required for cartilage formation and differently regulate joint development during skeletogenesis. I Bone Miner Res 17: 898-906. 


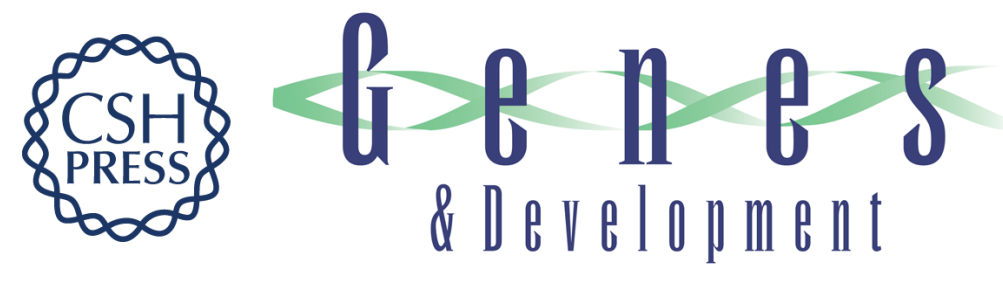

\section{$c$-Jun is required for the specification of joint cell fates}

Akinori Kan and Clifford J. Tabin

Genes Dev. 2013, 27:

Access the most recent version at doi:10.1101/gad.209239.112

Supplemental

Material

References

This article cites 31 articles, 10 of which can be accessed free at: http://genesdev.cshlp.org/content/27/5/514.full.html\#ref-list-1

\section{License}

Email Alerting Service

http://genesdev.cshlp.org/content/suppl/2013/03/08/27.5.514.DC1 right corner of the article or click here.

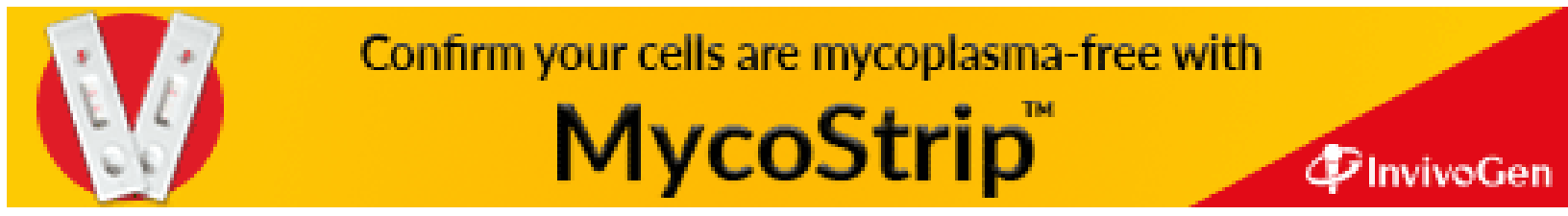

